\title{
Spherical Matching for Temporal Correspondence of Non-Rigid Surfaces
}

\author{
Jonathan Starck \& Adrian Hilton \\ Centre for Vision, Speech and Signal Processing \\ University of Surrey, Guildford, GU2 7XH, UK \\ j.starck,a.hilton@surrey.ac.uk \\ http://www.ee.surrey.ac.uk/CVSSP/VMRG
}

\begin{abstract}
This paper introduces spherical matching to estimate dense temporal correspondence of non-rigid surfaces with genus-zero topology. The spherical domain gives a consistent $2 D$ parameterisation of non-rigid surfaces for matching. Non-rigid $3 D$ surface correspondence is formulated as the recovery of a bijective mapping between two surfaces in the $2 D$ domain. Formulating matching as a $2 D$ bijection guarantees a continuous one-to-one surface correspondence without overfolding. This overcomes limitations of direct estimation of non-rigid surface correspondence in the $3 D$ domain. A multiple resolution coarse-to-fine algorithm is introduced to robustly estimate the dense correspondence which minimises the disparity in shape and appearance between two surfaces.

Spherical matching is applied to derive the temporal correspondence between non-rigid surfaces reconstructed at successive frames from multiple view video sequences of people. Dense surface correspondence is recovered across complete motion sequences for both textured and uniform regions, without the requirement for a prior model of human shape or kinematic structure for tracking.
\end{abstract}

\section{Introduction}

In this paper we address the problem of estimating spatio-temporal correspondence of non-rigid surfaces observed in multiple-view video sequences. Previous approaches to estimating non-rigid surface correspondence, or scene flow, directly match surfaces in 3D [4, 12, 14], which does not ensure a unique or continuous mapping for all surface points. We formulate the estimation of non-rigid correspondence between 3D surfaces with genus zero topology as 2D matching in the spherical domain. This guarantees a continuous bijective mapping between the 3D surfaces which does not overfold.

Spherical matching is applied to the recovery of dense correspondence from sequences of non-rigid 3D surface shape and appearance of people reconstructed from multiple view video. The reconstructed body shape is parameterised in the spherical domain by mapping a uniformly sampled surface onto the unit sphere while preserving the surface sampling rate. Parameterisation in the spherical domain provides a consistent representation for non-rigid matching irrespective of the body pose. Correspondence is then derived in the $2 \mathrm{D}$ spherical domain as a bijective mapping, a continuous one-to-one mapping for the entire surface. Compared to previous approaches based on 3D matching, formulating the problem as a bijection in the $2 \mathrm{D}$ domain guarantees a complete and continuous one-to-one match for a surface with no over-folding in the mapping.

This work makes the following contributions: (1) Spherical matching for the estimation of temporal correspondence between genus-zero surfaces parameterised on the 2D spherical domain; (2) A robust spherical matching algorithm that recovers 3D surface correspondence as a $2 \mathrm{D}$ bijective mapping, guaranteeing a continuous one-to-one surface correspondence with no over-folding; and (3) Recovery of dense non-rigid surface correspondence across complete motion sequences of people with correspondence recovered in regions of uniform appearance constrained by the global surface solution. Non-rigid correspondence is estimated without the requirement for a prior of model of human shape or kinematic structure.

\section{Related Work}

\subsection{Non-rigid Surface Correspondence}

Vedula et al. [14] introduced the concept of scene flow as the three-dimensional motion field of points in the world. Their work presented a framework for estimation of 3D non-rigid scene flow from the 2D optical flow estimated from multiple view image sequences. Space-time volumetric matching is employed to estimate temporal correspondence for volumetric reconstructions from multiple view 
video[14]. Carceroni et al. [4] estimate non-rigid spatiotemporal surface correspondence from multiple views using a dynamic surfel representation of local shape, reflectance and motion. Surfel sampling across space and time reconstructs the scene flow for points on the surface. These approaches estimate the temporal correspondence for nonrigid surfaces without prior assumptions on scene structure. Correspondence is estimated independently for each point on the surface resulting in noisy and erroneous flow field with potential for many-to-one matching. In contrast the approach of spherical matching introduced in this paper enforces a one-to-one temporal correspondence across the surface. This ensures spatial consistency across the surface without imposing prior models of non-rigid scene structure.

Previous approaches have been introduced to regularise estimates of non-rigid surface deformation across the surface. Variational methods have been employed to estimate regularised scene flow simultaneously across multiple views for a surface patch [12]. In principle this formulation extends to multiple views with arbitrary geometry but has only been demonstrated for stereo pairs with a single non-rigid surface. Neumann and Aloimanos[11] use a subdivision surface of spherical topology to directly estimate non-rigid spatio-temporal correspondence from multiple views. As with the approach presented in this paper both approaches use a multiple-resolution coarse-to-fine optimisation to estimate the dense temporal correspondence. Rather than direct surface matching in $\mathbb{R}^{3}$ our approach uses a spherical parameterisation of the surface to estimate correspondence on the surface of the sphere. This has the advantage of constraining the matching to a two-dimensional manifold and ensuring a one-to-one correspondence with the restriction that surface shape is of genus-zero topology.

\subsection{Surface Parameterisation}

Parameterisation of triangulated surface meshes in a regularly sampled domain has received considerable attention in computer graphics for efficient representation, remeshing, transmission and manipulation of object models [1]. Mesh parameterisation must satisfy the following properties: (1) bijective mapping - every vertex on the mesh surface in $\mathbb{R}^{3}$ has a unique one-to-one mapping to points in the domain space; (2) continuous mapping - adjacent vertices on the mesh map to adjacent points in the domain space. Parameterisation in a 2D planar domain space $\mathbb{R}^{2}$ requires the mesh to be topologically equivalent to a disk. Requiring dissection of the mesh into patches which are individually mapped to the planar domain to obtain a piecewise continuous mapping.

Spherical parameterisation allows genus-zero surfaces to be continuously parameterised in the spherical domain avoiding the requirement for mesh cuts. Recently robust techniques [13, 15] have been introduced for spherical parameterisation of genus-zero surfaces which include a wide variety of real objects. Spherical parameterisation allows the object surface to be resampled using uniform subdivision of simple polyhedra to form a regular mesh allowing smooth sub-division at multiple levels of detail, morphing between meshes, compression and shape analysis. In this paper we exploit spherical parameterisation to represent sequences of non-rigid objects reconstructed from multiple views. Spherical matching is introduced to establish the temporal correspondence between reconstructed object surfaces which guarantees a continuous, bijective mapping over the sequence. The spherical domain provides a natural parameterisation of objects such as people and allows shape and appearance matching to be performed across the entire object surface.

\subsection{Human Motion Reconstruction}

Reconstruction of human movement from single or multiple view image sequences has received considerable interest over the past decade [10]. Commonly, approaches assume prior knowledge of human anatomical structure and shape in the form of a model. Human motion estimation is typically performed in an analysis-by-synthesis framework by evaluating the fitness of a model pose against the image observations such as edges, silhouette, optic flow, feature matching, surface appearance or stereo points. In this paper we apply the spherical matching algorithm to photo-hull sequences of moving people reconstructed from multiple views. This approach estimates the dense temporal correspondence for the entire surface without prior assumptions on scene structure.

\section{Spherical Parameterisation}

In this section we outline the process of spherical parameterization based on previous research in computer graphics [13, 15]. Our starting point is a closed triangulated mesh $M$ in $\mathbb{R}^{3}$ of zero genus reconstructed from a single frame of multiple view video. We construct a map for $M$ onto the unit sphere $\hat{S},(M \rightarrow \hat{S})$, while minimizing the distortion of the representation in the spherical domain. The surface is then resampled onto a regular subdivision surface $S$ by constructing a map $(S \rightarrow \hat{S})$ such that we maintain the sampling density of the original mesh $M$ in resampling. Preservation of the surface sampling in parameterisation provides a consistent representation for matching and resampling provides a subdivision structure for multipleresolution matching as described in Section 4 Figure 1 illustrates the stages of spherical parameterisation and resampling. 


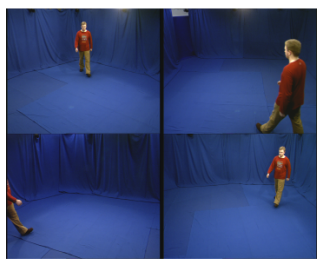

(a) Multiple view video

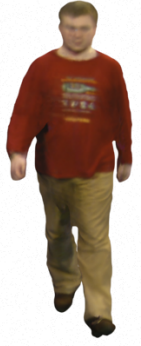

(b) $M$

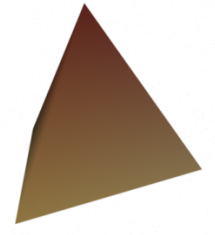

(c) $P M$

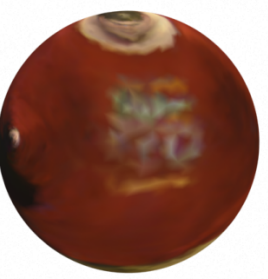

(d) $(P M \rightarrow \hat{S})$

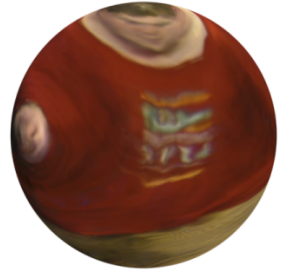

(e) $(S \rightarrow \hat{S})$

Figure 1. Stages of spherical parameterization: (a) multiple view capture; (b) reconstruction $M$; (c) progressive mesh $P M$ decimation to a tetrahedra; (d) embedding on the unit sphere $\hat{S}$; and (e) resampling onto a uniform subdivision surface $S$

\subsection{Surface reconstruction $M$}

Multiple view video sequences are captured in a dedicated 10 camera studio with a blue-screen backdrop for foreground segmentation. Cameras are positioned in a circle surrounding the centre of the studio, providing a capture volume of $3 \mathrm{~m} \times 2 \mathrm{~m} \times 2 \mathrm{~m}$. Sony DXC-9100P 3-CCD colour cameras are used, providing PAL-resolution progressive scan images. Intrinsic and extrinsic camera parameters are calibrated using a public domain calibration toolbox [3].

Volumetric reconstruction is performed, first by reconstructing the visual-hull from segmented foreground image silhouettes and then by applying the Generalized Voxel Coloring algorithm [6] to provide a colour consistent photohull. The surface of the volumetric reconstruction is extracted using the marching cubes algorithm [9]. The surface reconstruction is finally smoothed to provide a continuous representation for the surface normals. Surface colour is extracted at each vertex by blending colour samples from camera images using the hardware accelerated visibility test proposed by Debevec et al. [7]. The result of surface reconstruction is a uniformly sampled surface mesh $M$ as illustrated in Figure 1(b) with position, normal and colour attributes.

\subsection{Spherical mapping $(M \rightarrow \hat{S})$}

The map $(M \rightarrow \hat{S})$ is constructed using the coarseto-fine spherical parameterization technique introduced by Praun and Hoppe [13]. A mesh $M$ is simplified to a tetrahedron while creating a progressive mesh $P M$ structure [8]. The progressive mesh encodes a mesh $M$ as a base mesh, together with a sequence of vertex split operations that allow the mesh to be progressively refined back to $M$. Spherical mapping is achieved by first mapping the tetrahedron to the unit sphere, then traversing the progressive mesh sequence and inserting vertices onto the sphere maintaining the embedding in the spherical domain as illustrated in Figure 1.d).

For highly deformed genus-zero surfaces, producing a uniform sampling across all regions on the sphere is a challenging task. This represents a particular problem for the human body, where narrow limbs become highly distorted in the spherical domain. Praun and Hoppe [13] investigate several approaches to minimize distortion during mapping and perform mesh optimization to minimize the stretch in the representation with respect to the original mesh. In this work we seek a uniform sampling in the domain, preserving the sampling rate of the reconstructed surface. We propose a cost function derived from Zhou et al. [15] and optimise the spherical mesh to minimize the vertex sampling density on the sphere during mapping. This serves two purposes, it ensures an even vertex distribution to facilitate vertex insertion in the spherical domain and it minimizes vertex density to obtain a uniform sampling.

The sampling density $D\left(\underline{s}_{i}\right)$ at a spherical vertex $\underline{s}_{i}$ is defined as follows, where $i^{\prime}$ spans the 1-neighbourhood of a vertex $i, N_{i}$ is the valence of the vertex and $\Delta_{i i^{\prime}}$ is the spherical triangle with the directed edge $i i^{\prime}$ in the mesh.

$$
D\left(\underline{s}_{i}\right)=\frac{N_{i}}{\sum_{i^{\prime}} \operatorname{Area}\left(\Delta_{i i^{\prime}}\right)}
$$

We minimize the distortion in the vertex density on the sphere with respect to a target density $D_{0}$ using the following cost function, where $\nabla_{i i^{\prime}}$ represents the directed gradient on the edge $i i^{\prime}$.

$$
E_{\text {density }}(S)=\sum_{i} \sum_{i^{\prime}} \frac{D_{0}\left(\underline{s}_{i^{\prime}}\right)}{D\left(\underline{s}_{i^{\prime}}\right)}\left\|\nabla_{i i^{\prime}} \underline{s}_{i}\right\|^{2}
$$

For density minimization on the unit sphere we assume a uniform low resolution target density of, $D_{0}=1$. 


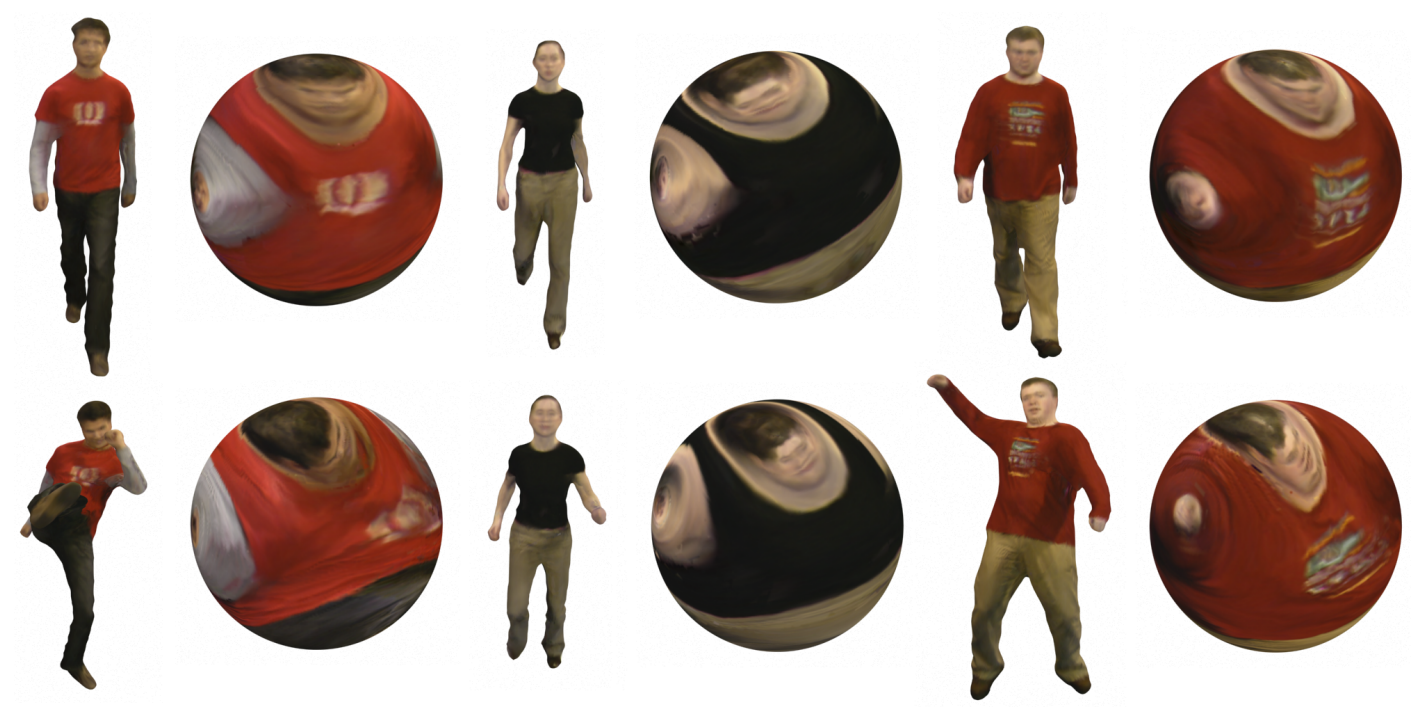

Figure 2. Result of spherical parameterisation for three subjects in different poses.

\subsection{Spherical remeshing $(S \rightarrow \hat{S})$}

Spherical mapping produces a highly distorted representation for the mesh $M$ in order to maintain the surface sampling in the mapping. This representation is unsuitable for deformation in spherical matching as the distorted vertex neighbourhoods restrict the space of feasible surface deformations without destroying the embedding on the sphere. The mesh is therefore regularly resampled on the spherical domain for deformation at multiple levels of detail in matching.

A subdivision surface $S$ is constructed using regular 1to-4 subdivision of a unit octahedron. We derive the mapping $S \rightarrow \hat{S}$ using the adaptive sampling scheme introduced by Zhou et al. [15]. The surface $S$ is updated to minimise the density cost function in Equation 2 with the target density derived from the surface $\hat{S}$. Gradient descent optimization corresponds to iterative application of the weighted laplacian operator $\Delta_{\text {density }}\left(\underline{s}_{i}\right)$ as proposed in [15].

$$
\Delta_{\text {density }}\left(\underline{s}_{i}\right)=\frac{1}{\sum_{i^{\prime}} \frac{D_{0}\left(\underline{s}_{i^{\prime}}\right)}{D\left(\underline{s}_{i^{\prime}}\right)}} \sum_{i^{\prime}} \frac{D_{0}\left(\underline{s}_{i^{\prime}}\right)}{D\left(\underline{s}_{i^{\prime}}\right)}\left(\underline{s}_{i}-\underline{s}_{i^{\prime}}\right)
$$

Given the mapping $M \rightarrow \hat{S}$ and of $S \rightarrow \hat{S}$, we can finally resample the position, normal and colour attributes of the original mesh $M$ onto the uniform domain of the subdivision surface $S$ as illustrated in Figure 1.e). Figure 2 shows the result of spherical mapping and remeshing for subjects in different poses.

\section{Spherical Matching}

In this section, we formulate surface to surface matching in $\mathbb{R}^{3}$ as a continuous mapping between two surfaces $\left(S \rightarrow S_{0}\right)$ in the spherical domain. Our approach poses the mapping problem as an energy minimization task. We construct a cost function for joint estimation of correspondence and deformation to minimize disparity in shape and appearance between two surfaces. A multiple-resolution coarseto-fine algorithm is presented to deform a surface to minimize the cost function while maintaining the embedding on the sphere and so ensuring a bijective mapping. The multiple-resolution approach using the subdivision structure $S$ enables large deformations for matching while maintaining the embedding in the spherical domain.

The matching function for minimization, $E_{\text {cost }}(S, \mathbf{W})$, is defined as a function of the set of vertices $S$ for a surface in the spherical domain and a fuzzy multiple-point correspondence matrix $\mathbf{W}=\left\{w_{i j} \in(0,1)\right\}$ defining the match between two surfaces $S=\left\{\underline{s}_{i}\right\}$ and $S_{0}=\left\{\underline{s}_{j}\right\}$. The function consists of three terms: $E_{\text {disparity }}$ measuring the distance in shape and colour of the surfaces; $E_{\text {deform }}$ measuring the distance between the surfaces in the spherical domain; and $E_{\text {regularise }}$ defining regularizing constraints on the deformation subject to a control parameter $\lambda$.

$$
\begin{aligned}
E_{\text {cost }}(S, \mathbf{W}) & =(1-\lambda) E_{\text {disparity }}(\mathbf{W}) \\
& +(1-\lambda) E_{\text {deform }}(S, \mathbf{W}) \\
& +\lambda E_{\text {regularise }}(S)
\end{aligned}
$$

In the following sections we describe each of the components of the matching functional followed by the algorithm 
for optimization and recovery of the final surface correspondence.

\subsection{Surface disparity}

Data fit is based on the similarity of a set of attributes defined on the surfaces $M$ and $M_{0}$. Spherical parameterization maps and samples a surface $M$ onto the spherical domain $S$. In energy minimisation we seek the correspondence $\mathbf{W}$ that minimises the disparity in shape and appearance using the position $\underline{x}$, surface normal $\underline{n}$ and RGB colour $\underline{c}$ of the resampled surfaces. The disparity is measured using a squared error metric and a separate energy function is constructed for each attribute as follows

$$
\begin{aligned}
E_{\text {disparity }}(\mathbf{W}) & =\frac{1}{\sigma_{x}^{2}} \sum_{i} \sum_{j} w_{i j}\left\|\underline{x}_{i}-\underline{x}_{j}\right\|^{2} \\
& +\frac{1}{\sigma_{n}^{2}} \sum_{i} \sum_{j} w_{i j}\left\|\underline{n}_{i}-\underline{n}_{j}\right\|^{2} \\
& +\frac{1}{\sigma_{c}^{2}} \sum_{i} \sum_{j} w_{i j}\left\|\underline{c}_{i}-\underline{c}_{j}\right\|^{2}
\end{aligned}
$$

The disparity terms are normalized by the expected variance $\sigma^{2}$ for a correct match. The variance parameters provide control over the expected range of disparities between two surfaces for each of the attributes used in matching.

\subsection{Spherical deformation}

The matching problem is posed as the deformation of the surface $S=\left\{\underline{s}_{i}\right\}$ to minimize the disparity with respect to the surface $S_{0}=\left\{\underline{s}_{j}\right\}$ in the spherical domain, $\underline{s} \in \mathbb{R}^{2}$. Surface deformation is driven by minimising the distance on the sphere based on the multiple-point correspondence $\mathbf{W}$ between the surfaces. In practise we linearize the problem and compute distances in $\mathbb{R}^{3}$ while constraining vertices to remain on the unit sphere. The deformation energy function is defined as

$$
\begin{aligned}
E_{\text {deform }}(S, \mathbf{W}) & =\frac{1}{T^{2}} \sum_{i} \sum_{j} w_{i j}\left\|\underline{s}_{i}-\underline{s}_{j}\right\|^{2} \\
& +\sum_{i} \sum_{j} w_{i j}\left(\log \left(w_{i j}\right)-1\right)
\end{aligned}
$$

A deterministic annealing approach [5] is adopted for optimization using an entropy term and temperature control parameter $T$ in the deformation functional. The term $w_{i j}$ defines a multiple-point fuzzy correspondence between the two surfaces and the temperature provides control of the correspondence during optimization. At a high temperature value the correspondence becomes more fuzzy and as the temperature is successively reduced the correspondence can be hardened, providing a coarse-to-fine refinement of the surface correspondence.

\subsection{Regularisation}

Regularization is used to minimize the distortion in the spherical domain while developing the map $S \rightarrow S_{0}$. Vertices $\underline{s}_{i}$ are updated sequentially and constrained to remain within the kernel of the 1-neighbourhood. This ensures that the surface $S$ maintains an embedding on the sphere. Left unconstrained the surface $S$ will quickly reach a local minima with vertices collapsed onto the border of the 1neighbourhood. Regularization is used to minimize the distortion in the distribution of vertices such that the surface $S$ can continue to deform to minimize the disparity function. This is achieved by minimizing the vertex density in the spherical domain during optimization

$$
E_{\text {regularise }}(S)=\frac{1}{T^{2}} E_{\text {density }}(S)
$$

A weighting parameter $\lambda$, Equation 4 is used to control the trade-off between regularization and data-fitting in optimization.

\subsection{Multi-resolution coarse-to-fine optimization}

Joint estimation of correspondence and deformation is solved using a strategy of alternately minimizing the matching cost with respect to the correspondence matrix $W$ and then updating the surface $S$ to satisfy the correspondence. For a fixed surface configuration $S$ a unique solution exists for the correspondence as follows. The constraint $\sum_{j} w_{i j}=1$ is imposed to enforce surface assignment $S \rightarrow S_{0}$.

$$
\begin{gathered}
w_{i j}^{\prime}=\exp \left(\frac{\left\|\underline{x}_{i}-\underline{x}_{j}\right\|^{2}}{\sigma_{x}^{2}}\right) \exp \left(\frac{\left\|\underline{n}_{i}-\underline{n}_{j}\right\|^{2}}{\sigma_{n}^{2}}\right) \\
\times \exp \left(\frac{\left\|\underline{c}_{i}-\underline{c}_{j}\right\|^{2}}{\sigma_{c}^{2}}\right) \exp \left(\frac{\left\|\underline{s}_{i}-\underline{s}_{j}\right\|^{2}}{T^{2}}\right) \\
w_{i j}=\frac{w_{i j}^{\prime}}{\sum_{j} w_{i j}^{\prime}}
\end{gathered}
$$

For a fixed correspondence $\mathbf{W}$ we use gradient descent to solve the continuous problem of surface deformation

$$
\frac{d E_{\text {cost }}}{d \underline{s}_{i}}=(1-\lambda) w_{i j}\left(\underline{s}_{i}-\underline{s}_{j}\right)+\lambda \Delta_{\text {density }}\left(\underline{s}_{i}\right)
$$

The optimization algorithm proceeds by first finding the global rotation that minimizes the cost function. The surface $S$ is Parameterised in terms of a rotation $(\theta, \phi, \psi)$ and 
the vertex positions $\underline{s}(\theta, \phi, \psi)$ are updated in parallel. A multiple-resolution strategy is then adopted for local optimization. Surface deformation is scheduled at successive resolutions of the subdivision surface $S$ starting with the base octahedron. Coarse-to-fine matching is achieved by setting the temperature parameter $T$ to restrict the range of matches $w_{i j}$ to lie principally within the kernel of the 1neighbourhood for a vertex. This is achieved using a value of $T$ equal to the edge length on the undeformed spherical mesh at each level of subdivision. Optimization at each resolution proceeds until the maximum update for a vertex reaches a fixed value $\varepsilon=0.0001$ on the unit sphere. The optimization algorithm terminates at the finest resolution of the subdivision surface $S$ and provides a bijection between the surfaces $S, S_{0}$.

\section{Application to Human Motion Tracking}

We apply spherical parameterization and matching to the problem of recovering temporal correspondence for the human body in multiple view image sequences. Surface reconstruction provides an independent and unstructured genus-zero surface mesh at each frame. Spherical matching is used to provide a continuous surface correspondence between successive frames.

For all multiple view video sequences we set the attribute variance parameters as follows, $\sigma_{x}=5 \mathrm{~cm}, \sigma_{n}=0.5\left(30^{\circ}\right)$, and $\sigma_{c}=50 \in(0,255)$. These values were derived using hand selected vertex correspondences in different motion sequences. The regularisation control parameter was fixed at $\lambda=0.75$. Originally, a locally adaptive regularisation parameter $\lambda\left(\underline{s}_{i}\right)$ was devised based on distortion of the vertex neighbourhood for $i$. In practise a constant value is sufficient as the regularisation term simply maintains an even vertex distribution such that the surface is free to deform and minimise the data fitting cost function. We perform optimization at 7 levels of subdivision for the base octahedron giving a final resolution of 65538 vertices.

Figure 2 shows the result of spherical parameterisation for three subjects in different body poses. In preserving the surface sampling in mapping to the unit sphere, we obtain a consistent distortion for the original surface sampling in the spherical domain and the spherical representation is consistent across a wide range of body poses.

Figures 3 and 4 demonstrate dense surface to surface matching for different motion sequences for three subjects. The figures show the path for a set of surface points in the first frame, tracked using frame-to-frame temporal correspondence through to the last frame in the sequence. Sequence lengths vary here from 25 to 45 frames. The results demonstrate that surface tracking is stable for complete motion sequences and that temporal correspondence is derived for regions of uniform shape and appearance where tech- niques based on optic flow would fail.

In comparison Figure 5 shows the result of non-rigid iterative closest point (ICP) [2] matching applied frame to frame for the motion sequences shown in Figure 4 Here the triangulated surface at each frame has been formulated as an elastically deformable object and iteratively updated to minimise the error at every vertex to the closest point match on the surface for the next frame. Matches recovered frame to frame drift across the sequence and correspondence is lost. Further constraints on surface deformation would be required to successfully recover correspondence in $3 \mathrm{D}$ matching to prevent the surface folding in mapping and ensure a one-to-one correspondence.

Spherical matching provides surface correspondence without the requirement for a prior parameterised model to constrain matching in 3D. The approach provides a continuous global solution for surface correspondence, a bijective mapping with no overfolding. This enables estimation of correspondence in regions of uniform shape and appearance, constrained by the global solution. The matching algorithm ensures a bijective mapping by maintaining the embedding for a mesh in the $2 \mathrm{D}$ spherical domain, with no vertex collapses or triangle flips during deformation. The multiple-resolution approach introduced enables large deformations during matching while maintaining this embedding.

The dense surface correspondence recovered using spherical matching is used to reconstruct articulated human motion. We use a skeletal model with 33 degrees of freedom posed by hand to match the first frame of a sequence. Skeletal pose is then derived to minimise the distance to the position of the tracked surface points using a modified Levenberg-Marquardt algorithm to solve the non-linear leasts-squares problem. Figures 3 and 4 show the pose of the skeletal model reconstructed from dense frame to frame surface matches. The correspondence derived frame to frame across an entire sequence enables recovery of the articulated body motion without use of a prior model for human motion tracking.

\section{Conclusions}

Spherical matching has been introduced to estimate dense temporal correspondence of genus-zero non-rigid surfaces. Surfaces are parameterised in the spherical domain providing a consistent representation for matching. A multiple-resolution coarse-to-fine algorithm has been developed which simultaneously optimises across the entire surface to estimate dense correspondence. Optimisation of surface-to-surface matching is performed with respect to disparity in surface shape, orientation and colour whilst maintaining a regular sampling. Spherical matching has three principal advantages for non-rigid temporal corre- 

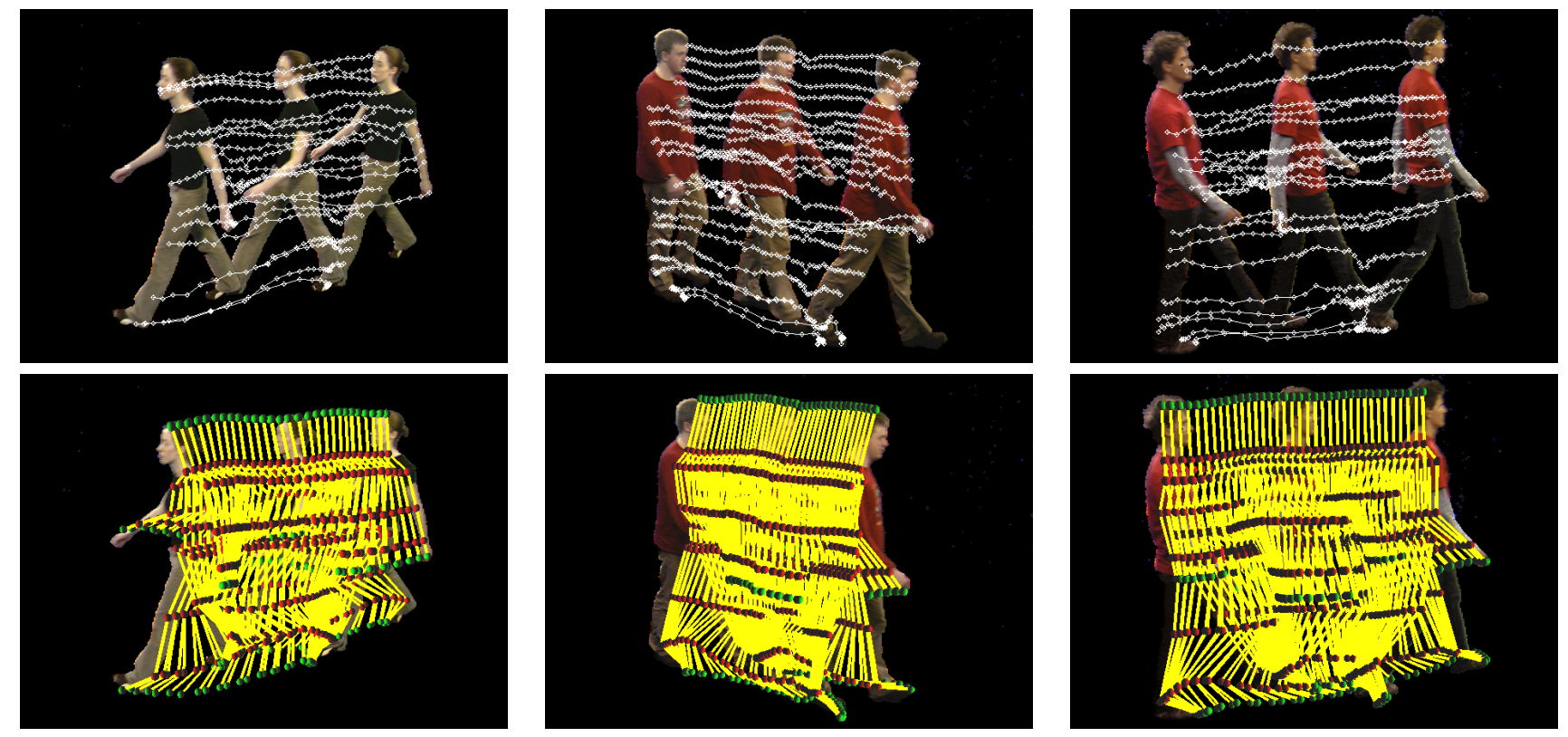

Figure 3. Surface correspondence and human motion reconstruction for walking sequences.
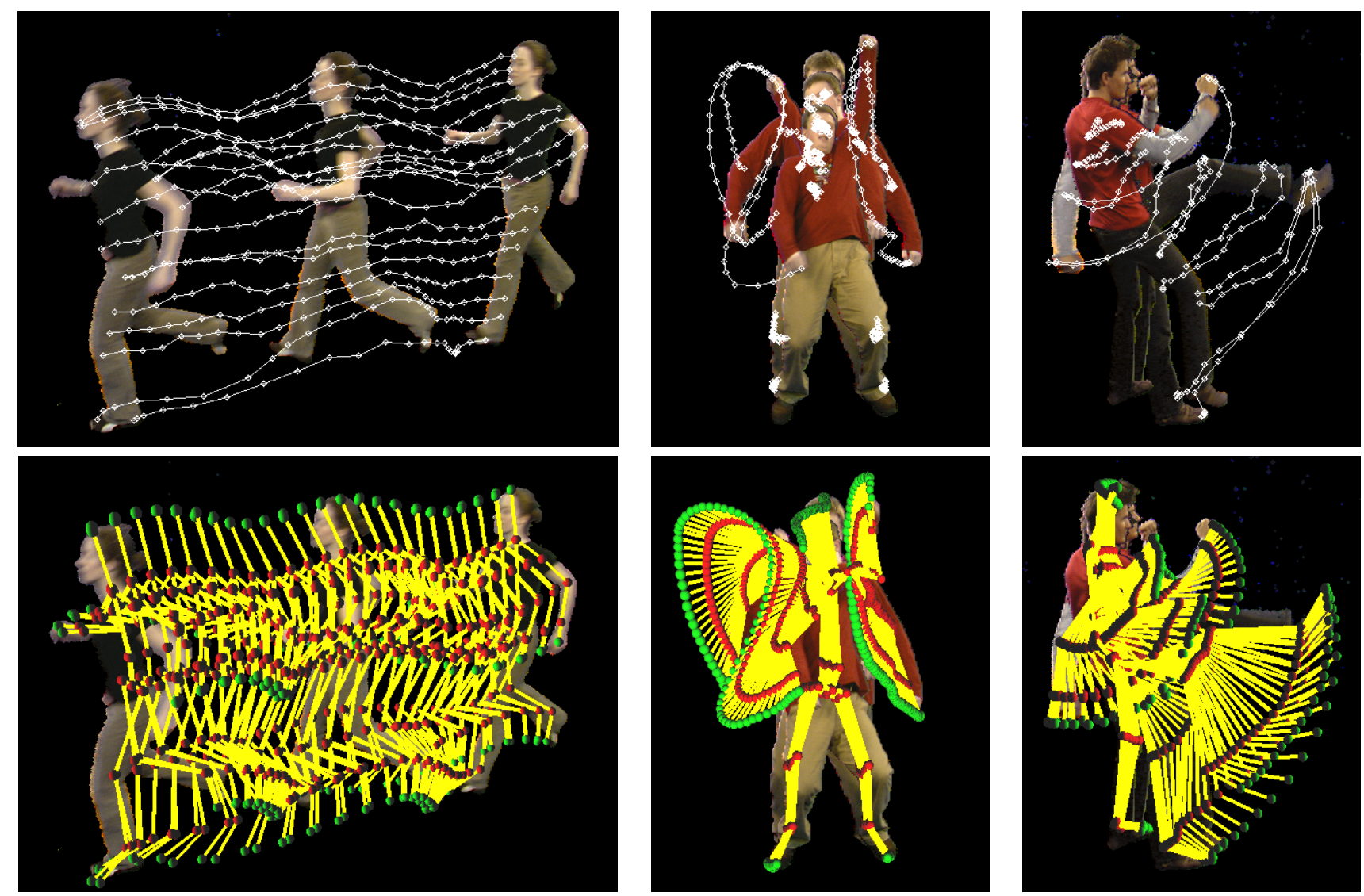

Figure 4. Surface correspondence and human motion reconstruction for jogging, falling and kicking sequences. 

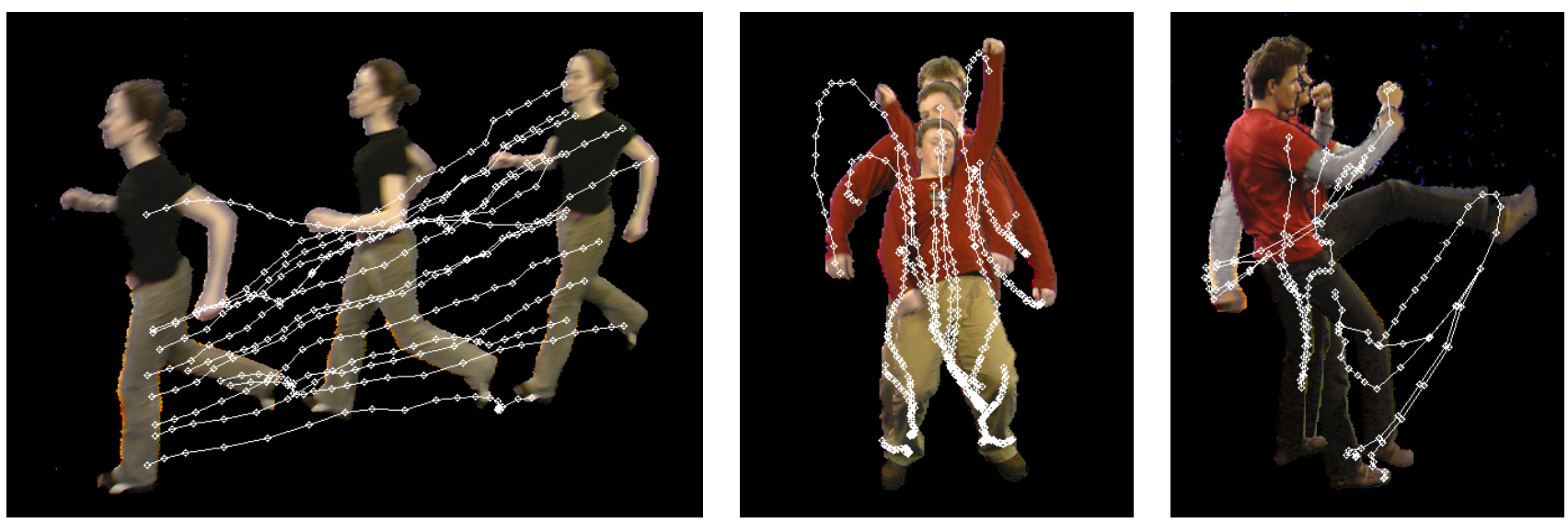

Figure 5. Comparison of suface correspondence esimated using non-rigid iterative closest point (ICP) matching. Frame to frame matches drift and correspondence is lost across a sequence.

spondence: (1) simultaneous optimisation across the entire surfaces including regions of uniform appearance; (2) bijective mapping providing a continuous one-to-one mapping between surfaces with no overfolding; and (3) estimation of dense temporal correspondence over non-rigid sequences. Spherical matching has been applied to multiple video of human movement. Dense temporal correspondence has been estimated for complete motion sequences. Spherical matching achieves frame-to-frame whole surface non-rigid alignment for complete sequences without the requirement for a prior model for human motion tracking.

\section{Acknowledgements}

This work was supported by EPSRC GR/S27962 Video Based Animation of People. The authors would like to thank Rachel Gartshore, Gregor Miller and Antonio Micilotta for volunteering to walk, run, kick and fall over all day.

\section{References}

[1] M. Alexa. Recent advances in mesh morphing. Computer Graphics forum, 21(2):173-197, 2002.

[2] P. Besl and N. McKay. A method for registration of 3-d shapes. IEEE Transactions on Pattern Analysis and Machine Intelligence, 14(2):239-256, 1992.

[3] J.-Y. Bouguet. Camera calibration toolbox for matlab: www.vision.caltech.edu/bouguetj/calib-doc. Technical report, MRL-INTEL, 2003.

[4] R. Carceroni and K. Kutulakos. Multi-view scene capture by surfel sampling: From video streams to non-rigid $3 \mathrm{~d}$ motion, shape and reflectance. International Journal of Computer Vision, 49(2-3):175-214, 2002.
[5] H. Chui and A. Rangarajan. A new point matching algorithm for non-rigid registration. Computer Vision and Image Understanding, 89:114-141, 2003.

[6] W. B. Culbertson, T. Malzbender, and G. Slabaugh. Generalized voxel coloring. Proceedings of the International Workshop on Vision Algorithms, Lecture Notes in Computer Science, 1883:100-115, 2000.

[7] P. Debevec, Y. Yu, and G. Borshukov. Efficient viewdependent image-based rendering with projective texturemapping. 9th Eurographics Rendering Workshop, pages 105-116, 1998.

[8] H. Hoppe. Progressive meshes. Proceedings ACM SIGGRAPH, pages 99-108, 1996.

[9] W. Lorenson and H. Cline. Marching cubes: A high resolution 3d surface construction algorithm. Computer Graphics, 21(4):163-169, 1987.

[10] T. Moeslund and E. Granum. A survey of computer visionbased human motion capture. Computer Vision and Image Understanding, 81(3):231-268, 2001.

[11] J. Neumann and Y. Aloimonos. Spatio-temporal stereo using multi-resolution subdivision surfaces. International Journal of Computer Vision, 47(1-3):181-193, 2002.

[12] J.-P. Pons, R. Keriven, O. Faugeras, and G. Hermosillo. Variational stereovision and $3 \mathrm{~d}$ scene flow estimation with statistical similarity measures. IEEE International Conference on Computer Vision, pages 597-602, 2003.

[13] E. Praun and H. Hoppe. Spherical parameterization and remeshing. Proceedings ACM SIGGRAPH, 2003.

[14] S. Vedula, S. Baker, P. Rander, R. Collins, and T. Kanade. Three-dimensional scene flow. IEEE Transactions on Pattern Analysis and Machine Intelligence, 27(3), 2005.

[15] K. Zhou, H. Bao, and J. Shi. 3d surface filtering using spherical harmonics. Computer-Aided Design,Elsevier, 36:363375, 2004. 